Published in final edited form as:

Mol Pharm. 2015 September 8; 12(9): 3399-3407. doi:10.1021/acs.molpharmaceut.5b00414.

\title{
Substrate-competitive activity-based profiling of ester prodrug activating enzymes
}

\author{
Hao Xu ${ }^{1,2}$, Jaimeen D. Majmudar ${ }^{3}$, Dahvid Davda ${ }^{4}$, Phani Ghanakota ${ }^{1}$, Ki H. Kim ${ }^{2}$, Heather

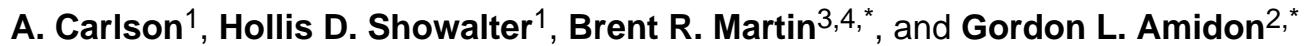 \\ 1 Department of Medicinal Chemistry, College of Pharmacy, University of Michigan, Ann Arbor, MI \\ 48109-1065, United States \\ 2 Department of Pharmaceutical Sciences, College of Pharmacy, University of Michigan, Ann \\ Arbor, Michigan 48109-1065, U.S.A. \\ ${ }^{3}$ Department of Chemistry, University of Michigan, 930 N. University Ave., Ann Arbor, MI 48109, \\ U.S.A. \\ 4Program in Chemical Biology, University of Michigan, 210 Washtenaw Avenue, 4008 Life \\ Sciences Institute, Ann Arbor, MI 48109-2216, U.S.A.
}

\section{Abstract}

Understanding the mechanistic basis of prodrug delivery and activation is critical for establishing species-specific prodrug sensitivities necessary for evaluating pre-clinical animal models and potential drug-drug interactions. Despite significant adoption of prodrug methodologies for enhanced pharmacokinetics, functional annotation of prodrug activating enzymes is laborious and often unaddressed. Activity-based protein profiling (ABPP) describes an emerging chemoproteomic approach to assay active site occupancy within a mechanistically similar enzyme class in native proteomes. The serine hydrolase enzyme family is broadly reactive with reporterlinked fluorophosphonates, which have shown to provide a mechanism-based covalent labeling strategy to assay the activation state and active site occupancy of cellular serine amidases, esterases, and thioesterases. Here we describe a modified ABPP approach using direct substrate competition to identify activating enzymes for an ethyl ester prodrug, the influenza neuraminidase inhibitor oseltamivir. Substrate-competitive ABPP analysis identified carboxylesterase 1 (CES1) as an oseltamivir-activating enzyme in intestinal cell homogenates. Saturating concentrations of oseltamivir lead to a 4-fold reduction in the observed rate constant for CES1 inactivation by

\footnotetext{
*Corresponding Authors Mailing Address: University of Michigan, Department of Chemistry, 930 N. University Ave., Ann Arbor, MI 48109. Tel. (734) 615-2867. brentrm @ umich.edu. University of Michigan, College of Pharmacy, 428 Church Street, Ann Arbor, MI 48109-1065. Tel.: (734) 764-2226. Fax: (734)763-6282. glamidon@umich.edu..

Author Contributions

H.X., D.D., G.L.A., and B.R.M. designed the experiments. H.X. and K.H.K. performed substrate-competitive ABPP experiments. H.X. and D.D. performed kinetic experiments; H.X., and J.D.M. performed proteomics analysis. P.G. and H.A.C. performed to the homology modeling work. H.X. and H.D.S. synthesized all the related compounds. H.X., D.D., and B.R.M. wrote the paper.

The authors declare no competing financial interest.

ASSOCIATED CONTENT

Supporting Information

Supplementary data, figures, tables, and extended experimental. This material is available free of charge via the internet at http:// pubs.acs.org.
} 
fluorophosphonates. WWL50, a reported carbamate inhibitor of mouse CES1, blocked oseltamivir hydrolysis activity in human cell homogenates, confirming CES1 is the primary prodrug activating enzyme for oseltamivir in human liver and intestinal cell lines. The related carbamate inhibitor WWL79 inhibited mouse, but not human CES1, providing a series of probes for analyzing prodrug activation mechanisms in different preclinical models. Overall, we present a substratecompetitive activity-based profiling approach for broadly surveying candidate prodrug hydrolyzing enzymes and outline the kinetic parameters for activating enzyme discovery, ester prodrug design and preclinical development of ester prodrugs.

\section{Keywords}

prodrug; ethyl ester hydrolysis; activation; activity-based protein profiling; SILAC; carboxylesterase

\section{INTRODUCTION}

Prodrug development typically begins with pre-clinical studies in mice, which are later extrapolated to predict efficacy in humans. Unfortunately, this process is complicated by interspecies differences in expression and significant genetic polymorphisms present across activating enzyme orthologues ${ }^{1}$. Early knowledge of specific activation pathways would accelerate this process, enabling direct assessment of species-specific prodrug activation pathways. While this is an important goal, traditional activity-guided biochemical fractionation and lengthy identification procedures hinder the annotation of prodrug activating enzymes. New methods for simplified identification of prodrug hydrolyzing enzymes would aid in the development, targeting, and optimization of new prodrug strategies.

The human genome encodes approximately 200 members of the serine hydrolase enzyme family, which carry out catalytic roles as amidases, esterases, and thioesterases of diverse cellular substrates ${ }^{2,3}$. Since many prodrug moieties involve esterification or amidation, it is not surprising that serine hydrolases are often implicated in the catabolism and activation of distinct prodrugs. As many of these enzymes remain unannotated, biochemical fractionation is the most common approach to identify candidate prodrug hydrolyzing enzymes. We previously used such methods to identify valacylovirase (BPHL) $)^{4}$, the activating enzyme for the orally absorbed antiviral prodrug valacyclovir. Astonishingly, BPHL was first identified 8 years after valacyclovir FDA approval, demonstrating the significant obstacles for mechanistic analysis of prodrug uptake pathways. Similarly, the prodrug activating enzymes for dabigatran etexilate were reported nearly 12 years after its initial pre-clinical discovery ${ }^{5}, 6$. With the annotation of the physiological prodrug activating enzymes, new opportunities arise to profile expression, activity, evolutionary conservation, and distinct polymorphisms that help design modes of delivery, optimize biodistribution, and effectively model pharmacokinetics. These examples highlight the present challenges in developing a mechanistic understanding of prodrug activation in drug delivery.

Activity-based protein profiling (ABPP) describes the use of reporter-linked covalent inhibitors to profile abundance, activation state, and the active-site occupancy across a 
mechanism-specific enzyme class. ABPP probes only react with functional and accessible reactive centers, and labeling is occluded by active site inhibitor occupancy. Since labeling is covalent, a reporter group (fluorophore, biotin, etc.) is irreversibly attached to its target enzymes, providing a chemical handle to visualize active enzymes using gel-based fluorescence, or assess active-site competitive occlusion by mass spectrometry-based proteomics. Importantly, activity-based profiling does not require purified enzymes or tailored substrate assays, enabling direct active-site interrogation in native lysates. Activitybased probes have been extensively developed for many enzyme families ${ }^{7}$, including serine hydrolases ${ }^{2,8}$, cysteine hydrolases ${ }^{9}$, and protein kinases ${ }^{10}$. In addition to profiling active hydrolases in complex proteomes, ABPP methods are especially useful for selectivityguided medicinal chemistry, where stepwise competitive profiling highlights off-targets for optimization based on both potency and selectivity in native proteomes ${ }^{11,12}$.

While competitive ABPP methods provide a robust approach for the discovery of active-site inhibitors, several considerations must be recognized to successfully profile targets of competitive, reversible inhibitors. Unlike competitive inhibitors, covalent inhibitors compete with activity-based probes in a non-equilibrium, time-dependent manner, which complicates profiling active site occupancy of moderate affinity ligands. To overcome this challenge, one solution is to weaken the potency of the ABPP probe using chemical modifications that preclude rapid enzyme inactivation. This kinetically-tuned competitive ABPP strategy was used to evaluate in vivo selectivity and tissue distribution of several reversible lysophospholipase inhibitors ${ }^{13}$.

Based on these findings, we sought to extend this methodology to proteome-wide substratecompetitive profiling. When the off-rate associated with initial substrate binding is much greater than the rate of enzyme catalysis, the $K_{\mathrm{m}}$ is roughly a measure of a substrate's binding affinity for the enzyme's active site. When more than one enzyme shares a common substrate, the enzyme with the highest substrate affinity is likely the first to reach active-site saturation. Once saturated, substrate turnover is restricted by the rate-determining enzymatic step characterized by the unimolecular rate constant $k_{\text {cat }}$. Therefore, we reasoned that timedependent, substrate-directed activity-based probe competition would allow identification of candidate prodrug activating enzymes with both reasonably low $K_{\mathrm{m}}$ and $k_{\text {cat }}$ values for a given prodrug. We recently demonstrated that high concentrations of the ester prodrug enalapril sufficiently compete for active site occupancy and impede fluorophosphonate inactivation of recombinant CES $1{ }^{14}$. Given these steady-state kinetic parameters, the success of this method relies on rapid incubation times (seconds to a few minutes) and optimized probe concentrations, as an irreversible active site probe will react to completion and eventually eliminate any observable differences ${ }^{13,14}$.

Using this approach, we demonstrate that saturating concentrations of the ethyl ester prodrug oseltamivir reduced fluorophosphonate labeling of specific targets in cellular homogenates.

Human liver carboxylesterase 1 (CES1) is a known activating enzyme of several ester prodrugs, including oseltamivir ${ }^{5,15-18}$. The co-expression of multiple carboxylesterase (CES) genes ${ }^{19,20}$ and the differential substrate preferences between species complicates direct analysis of contributions from distinct CES enzymes. To address this issue, we profiled a subset of reported mechanism-based covalent mouse CES1 inhibitors, 4-fluoro-3- 
methylphenyl cyclohexylcarbamate (WWL50) and phenyl (3-morpholinopropyl)carbamate $\left(\right.$ WWL79) ${ }^{21}$. Quantitative mass spectrometry and kinetic assays confirmed WWL50 potently inactivates CES1, CES2, and the highly related, human-specific carboxylesterase, CES1P1. Interestingly, WWL79 only inhibits mouse CES1, and has little effect on human CES1, which we extrapolate from homology modeling to result from a more restrictive and hydrophobic active site. Therefore, the CES1 inhibitors WWL50 and WWL79 are useful tools for defining species-specific CES1 ester prodrug activation pathways. Overall, we demonstrate substrate-competitive ABPP can be used to quickly identify substrate-enzyme pairs in the context of a complex proteome, opening new opportunities to couple prodrug development with mechanistic analysis of enzymatic activation pathways.

\section{EXPERIMENTAL SECTION}

\section{Materials}

Oseltamivir phosphate (Allichem), enalapril maleate salt (Sigma-Aldrich), tazarotene (Selleckchem), benazepril $\mathrm{HCl}$ (Selleckchem), human carboxylesterase 1b (Corning), and carboxylesterase 2 (corning) were purchased from commercial sources. FP-PEG-TAMRA, FPPEG-biotin and FP-TAMRA were prepared as previously described ${ }^{14,22}$. WWL50 and WWL79 were synthesized according to reported methods (Supplementary Methods).

\section{Cell culture, lysis, and fractionization}

The human colon carcinoma cell line Caco-2 (from ATCC, HTB-37) and human liver hepatocellular carcinoma cell line HepG2 (from ATCC, HB-8065) were cultured separately in complete medium (DMEM with 1\% nonessential amino acid and 10\% FBS) in $100 \mathrm{~mm}$ tissue culture dishes in an atmosphere of $5 \% \mathrm{CO}_{2}$ at $37{ }^{\circ} \mathrm{C}$. Cells were grown 13-14 days post-confluence and collected in ice-cold $50 \mathrm{mM}$ Tris buffer ( $\mathrm{pH}$ 7.4) for immediate dounce homogenization, followed high speed $(100,000 \times \mathrm{g}$ for $45 \mathrm{~min})$ to separate soluble $(\mathrm{S} 100)$ and insoluble (P100) fractions. Protein sample concentrations were normalized using the BCA assay and stored at $-80^{\circ} \mathrm{C}$ before use.

\section{Prodrug hydrolysis assays}

Caco-2 S100 and P100 fractions (1 mg/mL) in $50 \mathrm{mM}$ Tris buffer $(\mathrm{pH} 7.4)$ were treated with DMSO (0.5\%) as control, FP-PEG-TAMRA (4 $\mu \mathrm{M}$ or as noted in figures), WWL50 (10 $\mu \mathrm{M})$, or WWL79 $(10 \mu \mathrm{M})$ for 30 minutes at room temperature. Prodrugs were added for the indicated times and the reactions were terminated with two volume equivalents of acetonitrile (ACN) with $0.1 \%$ TFA. Precipitated proteins were removed by centrifugation $(13,200 \times \mathrm{g}$ for $3 \mathrm{~min})$ at room temperature, and the supernatant collected and added to Microplate PVDF filter. The filtrates were injected using an Agilent 1100 HPLC system onto a $4.6 \times 150 \mathrm{~mm} 3.5$ micron ZORBAX Eclipse XDB-C18 column (Agilent) and separated with an 11 min gradient from $2 \%$ - 90\% buffer B (Buffer A: $\mathrm{H}_{2} \mathrm{O}$ with $0.1 \%$ TFA; Buffer B: ACN with $0.1 \%$ TFA). Products were monitored by UV absorption (220 nm for oseltamivir and enalapril, $254 \mathrm{~nm}$ for other prodrugs). Hydrolysis rates were calculated by integrating the relative areas under the curve. 
CES1 transient kinetic analysis-Recombinant human carboxylesterase $1(100 \mathrm{nM}$, $6.25 \mathrm{mg} / \mathrm{L}$ ) was incubated with oseltamivir $(10 \mathrm{mM})$ or vehicle (DMSO) for $8 \mathrm{~min}$ at room temperature in TBS-F127 buffer (50 mM Tris, $150 \mathrm{mM} \mathrm{NaCl}, 0.5 \mathrm{~g} / \mathrm{L}$ Pluronic F127, pH 7.4), followed by the addition of FP-PEG-TAMRA $(1 \mu \mathrm{M})$. At the indicated time points, 20 $\mu \mathrm{L}$ of reaction mixture was taken and inactivated with $4 \mu \mathrm{L}$ of $6 \times$ Laemmli sample buffer and heated at $85^{\circ} \mathrm{C}$, for $5 \mathrm{~min}$. Mixtures were separated by SDS-PAGE using a $4 \%-20 \%$ precast Tris-Glycine gel (Invitrogen) and imaged using a Typhoon 9200 fluorescence scanner. The fluorescence intensity of each band was quantified using ImageJ and data fitting was performed in GraphPad Prism 6. Non-linear regression analysis was used to fit the data to a first-order exponential curve of the form $F(t)=F_{\text {final }} *\left(1-\exp \left(-k_{o b s} * t\right)\right.$, where $F(t)$ is the fluorescence intensity at a given time, $k_{\mathrm{obs}}$ is the observed pseudo-first order rate constant and $t$ is reaction time.

\section{CES1 steady-state kinetic analysis}

Purified recombinant human carboxylesterase $1(160 \mathrm{nM}, 10 \mathrm{mg} / \mathrm{L})$ was incubated with varying concentrations of oseltamivir $(0.2-5 \mathrm{mM})$ in Tris buffer $(50 \mathrm{mM}, 100 \mu \mathrm{g} / \mathrm{ml} \mathrm{BSA}$, $\mathrm{pH}$ 7.4). A sample of the reaction mixture $(30 \mu \mathrm{L})$ was removed at $0,5,10,15$ and $20 \mathrm{~min}$ post initiation and quenched in $60 \mu \mathrm{L}$ of acetonitrile with $0.1 \%$ TFA. Mixtures were separated on an HPLC (Agilent 1100) equipped with a $4.6 \times 150 \mathrm{~mm} 3.5$ micron ZORBAX Eclipse XDB-C18 column using an acetonitrile gradient (11 min gradient from 2\% - 90\%). Relative levels of substrate and product were calculated by integrating the corresponding chromatographic peaks. The rate of product formation at each substrate concentration was obtained by measuring the slope associated with the time-course and data fitting was performed in GraphPad Prism 6. Non-linear regression analysis was used to fit the data to the steady-state Michaelis-Menten equation.

\section{Substrate-competitive activity-based protein profiling}

Oseltamivir competition assays were performed by first incubating proteome aliquots ( 1 $\mathrm{mg} / \mathrm{mL}$ final) with oseltamivir $(10 \mathrm{mM})$ for $10 \mathrm{~min}$ at room temperature in $50 \mathrm{mM}$ Tris buffer (pH 7.4). FP-PEG-TAMRA was then added at room temperature for less than 5 minutes and quenched with sample loading buffer at $85^{\circ} \mathrm{C}$ for $5 \mathrm{~min}$, followed by SDSPAGE analysis (4\% - 20\%, Tris-Glycine Pre-cast gel (Invitrogen)) and visualized on-gel in a Typhoon 9200 fluorescence imager. For competitive activity-based selectivity assays, proteome samples $(1 \mathrm{mg} / \mathrm{mL}$ in $50 \mathrm{mM}$ Tris buffer, $\mathrm{pH}$ 7.4) were incubated with WWL79 or WWL50 at varying concentrations for $30 \mathrm{~min}$ at room temperature, followed by addition of FP-PEG-TAMRA ( $4 \mu \mathrm{M})$ for 30 minutes, separated by SDS-PAGE, and analyzed by ingel fluorescence analysis.

\section{ABPP-SILAC analysis}

Caco-2 cells were passaged more than 6-times in SILAC-DMEM (Thermo) supplemented with either $100 \mu \mathrm{g} / \mathrm{ml}$ of ${ }^{12} \mathrm{C}_{6}{ }^{14} \mathrm{~N}_{2}$-lysine and ${ }^{12} \mathrm{C}_{6}{ }^{14} \mathrm{~N}_{4}$-arginine (LIGHT condition) or ${ }^{13} \mathrm{C}_{6}{ }^{15} \mathrm{~N}_{2}$-lysine and ${ }^{13} \mathrm{C}_{6}{ }^{15} \mathrm{~N}_{4}$-arginine (HEAVY condition), $10 \%$ dialyzed FBS (JR Scientific), and 1x penicillin/streptomycin (Life Technologies). Fractionated lysates were adjusted to final protein concentration of $1.5 \mathrm{mg} / \mathrm{mL}$. Light and heavy fractions $(1.5 \mathrm{~mL}$ 
each) were incubated separately with WWL50 $(15 \mu \mathrm{M})$ or vehicle for $30 \mathrm{~min}$, followed by addition of FP-PEG-biotin $(7.5 \mu \mathrm{M})$ for 1 hour. Light and heavy proteomes were then mixed in a 1:1 ratio and precipitated by chloroform: methanol extraction $\left(1.5 \mathrm{vol} \mathrm{CHCl}_{3}: 4 \mathrm{vol}\right.$ $\mathrm{MeOH}: 3$ vol water), briefly vortexed, and centrifuged $(10,000 \times \mathrm{g})$ at room temperature for 10 min to separate the aqueous and organic phases. The top aqueous phase was carefully discarded, followed by addition of an additional 3 volumes of methanol and centrifugation $(10,000 \times \mathrm{g}, 10 \mathrm{~min})$. The resulting protein precipitate was then mixed with 4 volumes of methanol $(6 \mathrm{~mL})$ and sonicated at $4{ }^{\circ} \mathrm{C}$ until homogenous. The protein precipitate was centrifuged $(10,000 \times \mathrm{g}, 10 \mathrm{~min})$, decanted, air-dried for 2-3 min, and re-solubilized in 500 $\mu \mathrm{L}$ of $6 \mathrm{M}$ urea / $50 \mathrm{mM}$ ammonium bicarbonate buffer. The sample was then reduced with DTT $\left(10 \mathrm{mM}, 65^{\circ} \mathrm{C}, 15 \mathrm{~min}\right)$ and alkylated with iodoacetamide (40 mM, room temperature, $30 \mathrm{~min}$, in dark). Next, $140 \mu \mathrm{L}$ of $10 \%$ SDS was added and the protein mixture was incubated at $65{ }^{\circ} \mathrm{C}$ for $10 \mathrm{~min}$., followed by dilution with $5.5 \mathrm{~mL}$ of PBS buffer. Once cooled, streptavidin-agarose resin (Thermo Scientific, 50\% slurry, $200 \mu \mathrm{L}$ ) was added and rotated at room temperature for 1.5 hour. After binding to streptavidin beads, the supernatant was removed and the resin was washed three times with $1 \%$ SDS in PBS buffer $(200 \mu \mathrm{L})$ and three times with PBS buffer $(200 \mu \mathrm{L})$. On-bead digestion was performed for $12 \mathrm{~h}$ at 37 ${ }^{\circ} \mathrm{C}$ with trypsin (Promega) in the presence of $\mathrm{CaCl}_{2}(2 \mathrm{mM})$. Peptide samples diluted to a final concentration of 30-50 ng / $\mu \mathrm{L}$ with $5 \%(\mathrm{v} / \mathrm{v})$ formic acid.

\section{Mass Spectrometry and Data Analysis}

Tryptic digests were separated using a Waters NanoAcquity UPLC system equipped with a $5 \mu \mathrm{m}$ Symmetry $\mathrm{C}_{18}(180 \mu \mathrm{m} \times 20 \mathrm{~mm})$ trap column and a $1.8 \mu \mathrm{m}$ High Strength Silica (HSS-T3) analytical column $(75 \mu \mathrm{m} \times 150 \mathrm{~mm})$ heated to $35^{\circ} \mathrm{C}$ coupled to a picotip emitter (New Objective). Tryptic peptides were loaded onto the trap column over 3 minutes, followed by analytical separation over a 90 minute gradient (3\% acetonitrile to $40 \%$ acetonitrile over 90 minutes). Peptides were analyzed using a Waters Synapt G2S HDMS time-of-flight mass spectrometer with ion mobility separation and data independent fragmentation algorithms. The quadruple mass analyzer was manually set for mass 500, 600 and 700. The sampling cone was adjusted to $32 \mathrm{eV}$ and the nano flow gas was set to flow at 0.2 bar. The purge gas was set to flow at $50 \mathrm{~L} / \mathrm{h}$ and the source temperature was set at 70 ${ }^{\circ} \mathrm{C}$. For all measurements, the mass spectrometer was operated in V-mode (resolution mode) with a resolving power of at least 20,000 FWHM (full width at half maximum) in positivemode ESI. The time-of-flight analyzer of the mass spectrometer was calibrated with a 100 fmol / $\mu \mathrm{L}$ solution of [Glu1]-Fibrinopeptide B from $\mathrm{m} / z 50$ to 1250 to within $0.5 \mathrm{ppm}$. The data was corrected in post-acquisition analysis using the doubly charged monoisotopic ion of [Glu1]-Fibrinopeptide B $(\mathrm{m} / \mathrm{z}=785.8426)$ collected every 30 seconds from a separate calibrant fluidics source coupled to a tapertip emitter (New Objective). Accurate mass data was collected in data-independent acquisition (DIA) mode in combination with in-line ion mobility separation (IMS). For IMS, the wave height was set as $40 \mathrm{~V}$ and IMS wave velocity as $600 \mathrm{~m} / \mathrm{s}$. The spectral acquisition time in each mode was $0.5 \mathrm{~s}$. In low-energy MS mode, data were collected without applying collision energy in the trap or the transfer stage. A collision energy (CE) ramp from $15 \mathrm{eV}$ to $45 \mathrm{eV}$ during each 0.5 s-integration was used as standard setting for the elevated energy MS scan in the transfer region for $\mathrm{HDMS}^{\mathrm{E}}$ mode. LC-MS spectra were collected in continuum mode and searched using the ProteinLynx 
Global SERVER version 3.0.2 (Waters) against the reviewed human reference proteome (UniProtKB downloaded on 2014-08-01). Precursor- and fragment-ion mass tolerances were automatically determined by PLGS 3.0.2 based on the following search criteria: (i) trypsin as digestion enzyme, (ii) a maximum of one missed cleavage, (iii) lysine (+8) or arginine $(+10)$ defined as fixed modifier reagent groups, (iv) carbamidomethyl cysteine as a fixed modification and methionine oxidation as the variable modification, (v) a minimum of two identified fragment ions per peptide and a minimum of five fragments per protein, and (vi) at least two identified peptides / protein. The false discovery rate (FDR) for peptide and protein identification was set at $1 \%$ using a reversed database. Using in-house Python scripts, all data from technical and biological replicates were merged, removing any precursors greater than $\pm 10 \mathrm{ppm}$ in mass difference from the calculated theoretical mass. SILAC ratios were computed based on MS1 intensities of the SILAC precursor pairs. All reported ratios in the manuscript are consolidated ratios from runs in each direction and from merging technical replicates.

\section{Homology Modeling and Covalent Docking}

The homology model of mouse CES1 was built using chain A of the crystal structure of human CES1 (PDB ID: 2H7C) as a template. 2H7C has the highest resolution (2£) of all the available human CES1 crystal structures. The homology model was built using Prime P $^{23,24}$ from the Schrodinger software suite. To prepare human and mouse CES1s for covalent docking, each protein was processed with the Protein Preparation Wizard to add hydrogens and address histidine tautomers and asparagine and glutamine flips where necessary. The carbamate bond to be broken in WWL50 and 79 for covalent docking was specified using a SMARTS string. The shape and volume of the ligand free binding site was generated with the program HOLLOW ${ }^{25}$ using a grid spacing of $0.25 \AA$ and covalently docked models along with the volume of the binding site were rendered using PyMOL.

\section{RESULTS}

\section{Substrate-competitive profiling of oseltamivir-binding serine hydrolases}

Ethyl ester hydrolysis of oseltamivir is necessary to liberate the active carboxylate essential for neuraminidase inhibition ${ }^{26}$ (Figure 1A). CES1 has been previously biochemically characterized as an oseltamivir-activating enzyme, yet human CES1 polymorphisms do not account for all of the variability in patient prodrug activation ${ }^{27}$. Under the background of this model, we sought to establish if substrate-competitive activity-based profiling could directly annotate oseltamivir-activating enzymes among serine hydrolases present in human Caco- 2 cell homogenates, a cell line that expresses multiple carboxylesterase ${ }^{28}$ and is widely used for modeling human intestinal absorption ${ }^{29}$. Using this system, FP-PEGTAMRA completely blocked oseltamivir activation, confirming members of the serine hydrolase enzyme family are solely responsible for ethyl ester hydrolysis (Figure 1B).

Next, Caco-2 soluble and insoluble homogenates were incubated with excess oseltamivir along with time-dependent addition of FP-PEG-TAMRA ${ }^{11}$, which enables in-gel analysis of serine hydrolase activities using a flatbed fluorescence gel scanner. After one minute of competition, inhibition was detectable for both a $30 \mathrm{kD}$ and $60 \mathrm{kD}$ enzyme (Figure 1C). 
Both activities partitioned with the soluble (S100) (Figure 1D) and insoluble (P100) proteome fractions (Figure 1E). Interestingly, the $60 \mathrm{kD}$ enzyme, but not the $30 \mathrm{kD}$ enzyme, lost time-dependent competitive inhibition after 1 minute. In addition, higher FP-PEGTAMRA concentrations reduced oseltamivir competition for the $60 \mathrm{kD}$ enzyme (Supplementary Figure 1), but had little effect on the $30 \mathrm{kD}$ enzyme. Taken together, these data demonstrates that high concentrations of oseltamivir can compete for active site occupancy with the ABPP probe FP-PEG-TAMRA in a complex proteome. Furthermore, this assay identifies targets likely inhibited by oseltamivir ( $30 \mathrm{kD}$ enzyme) as well as candidate activating enzymes (60 kD enzyme). Importantly, such rapid fluorophosphonate labeling reduces the profile of detectable targets, biasing the profile towards abundant or highly FP-reactive enzymes.

\section{Oseltamivir is hydrolyzed by CES1}

CES1 was previously reported as an oseltamivir hydrolyzing enzyme, and likely corresponds to the $60 \mathrm{kD}$ molecular weight enzyme identified by gel-based substratecompetitive ABPP. In order to validate the $60 \mathrm{kD}$ enzyme as CES1, we synthesized two recently reported mouse CES1 covalent inhibitors, WWL50 and WWL79 for further analysis (Figure 2A). Each inhibitor was added at room temperature to Caco-2 homogenates for 30 minutes, followed by FP-PEGTAMRA labeling for an additional 30 minutes. Gelbased competitive ABPP analysis revealed complete inhibition of the $60 \mathrm{kD}$ enzyme by WWL50, but essentially no effect by WWL79 (Figure 2B). Neither inhibitor affected the 30 $\mathrm{kD}$ band shown to be sensitive to oseltamivir. Additionally, WWL50 is nearly 100-times more potent towards human CES1 $\left(\mathrm{IC}_{50}=4.3 \pm 0.6 \mathrm{nM}\right)$ than human CES2 $\left(\mathrm{IC}_{50}=429.0 \pm\right.$ $2.6 \mathrm{nM}$ ) (Figure 2C).

While WWL50 appears highly selective by gel-based competitive ABPP, we profiled selectivity against other less abundant WWL50 targets by mass spectrometry. Using stable isotope labeling by amino acids in cell culture (SILAC), Caco-2 cells were labeled with media supplemented with either normal or isotopically-heavy (Lys8, Arg10) amino acids for several passages. After separate treatment with either DMSO or WWL50, SILAC pairs of soluble and insoluble homogenates were labeled with FP-PEG-biotin and mixed in a 1:1 ratio. Following streptavidin enrichment and trypsin digestion, eluted tryptic peptides were analyzed by high-resolution mass spectrometry to quantify targets of WWL50 inhibition ${ }^{30}$. Any hydrolase inactivated by WWL50 is unreactive with fluorophosphonate-linked biotin, reducing streptavidin enrichment in one of the paired samples. This difference is measured by quantifying the ion peak area for each hydrolase peptide in both the isotopically light and heavy samples, generating a relative enrichment ratio correlated with WWL50 inhibition. Of the 41 quantified hydrolases in Caco-2 cells, WWL50 exclusively blocked the enrichment of CES1, CES2, and CES1P1 (Figure 3 and Supplementary Tables 1-2). Summed across reciprocal replicates (DMSO-Light/WWL50-Heavy and WWL50-Light/DMSO-Heavy), CES1 had 138 quantified SILAC peptide pairs, while CES1P1 had 15, and CES2 had just 6, signifying the relative abundance of each enzyme. Based on these findings, CES1 is the predominant WWL50 target in Caco-2 cells contributing to oseltamivir hydrolysis. 
Next, the contribution of CES1 to oseltamivir hydrolysis was measured in Caco-2 or liver HepG2 cell homogenates treated with carboxylesterase inhibitors (Figure 4 and 5A). Hydrolytic activity was roughly similar in both soluble (S100) and insoluble (P100) fractions, tracking with CES1 partitioning. Incubation with either FP-PEG-TAMRA or WWL50 completely abolished oseltamivir hydrolysis activity (>95\%), while WWL79 showed fractional inhibition in Caco-2 homogenates. Collectively, these data confirm that CES1 is the primary oseltamivir hydrolase in HepG2 and Caco-2 homogenates, excluding contributions from the oseltamivir-sensitive $30 \mathrm{kD}$ enzyme observed by substratecompetitive ABPP. Similarly, activation of the ester prodrugs enalapril, benazepril, and tazarotene was also eliminated by WWL50 in both soluble (S100) (Figure 5D) and insoluble (P100) fractions (Figure 5E). Importantly, valacyclovir activation was unaffected by WWL50, as the primary activating enzyme is valacyclovirase (BPHL), which is not inhibited by WWL50 or FP-PEG-TAMRA.

\section{Kinetic parameters for substrate-competitive ABPP}

As the primary activating enzyme of oseltamivir, CES1 is a promising model to define the kinetic parameters necessary for profiling prodrug-activating enzymes by substratecompetitive ABPP. Steady-state kinetic analysis of CES1-catalyzed oseltamivir hydrolysis was performed by measuring the rate of product formation in a discontinuous chromatographic assay. At a given substrate concentration, samples from the reaction mixture were taken at specific intervals and were subjected to HPLC separation and quantified by UV absorbance. CES1 showed surprisingly poor activity with oseltamivir, with $K_{\mathrm{M}}$ and $k_{\text {cat }}$ values determined as $2.9 \times 10^{-3} \mathrm{M}$ and $0.75 \mathrm{~s}^{-1}$ respectively. The calculated catalytic efficiency $\left(k_{\text {cat }} / K_{\mathrm{M}}\right)$ value is $258.6 \mathrm{~s}^{-1} \cdot \mathrm{M}^{-1}$, defining oseltamivir as a relatively poor substrate for CES1 (Figure 6A), yet not uncommon for enzymes operating in secondary metabolism ${ }^{31}$. Despite these obstacles, substrate-competitive occupancy is still observed under saturating conditions. In this case, the slow $k_{\text {cat }}$ likely enhances competition by extending substrate active site residency time.

In order to confirm substrate-competitive fluorophosphonate inactivation, time-dependent assays were performed using in-gel fluorescence of FP-PEG-TAMRA labeled CES1 (Figure 6B and Supplementary Figure S2). In the presence of excess fluorophosphonate, the timedependent labeling of recombinant CES1 by FP-PEG-TAMRA followed pseudo-first-order reaction kinetics with an observed rate constant $\left(k_{o b s}\right)$ of $4 \mathrm{~s}^{-1}$, yielding an inactivation rate constant $\left(k_{\text {inact }}=k_{\text {obs }} /[\mathrm{I}]\right)$ of $4.0 \times 10^{6} \mathrm{~s}^{-1} \cdot \mathrm{M}^{-1}$. This value that is 15,000 -times larger than the pseudo-second-order rate constant of CES1 for oseltamivir $\left(k_{\mathrm{cat}} / K_{\mathrm{M}}=258.6 \mathrm{~s}^{-1} \cdot \mathrm{M}^{-1}\right)$. Given the rapid $k_{\text {inact }}$ value, fluorophosphonate-labeled CES1 fluorescence was detectable after 5 seconds, and nearly saturated by 1 minute. We envisioned that under fully saturating substrate conditions, the rate of fluorophosphonate inactivation is limited by the rate at which CES1 clears its active site by turning over oseltamivir, represented by $k_{\text {cat }}$. Thus, at oseltamivir saturation, the maximum theoretical reduction in the observed rate constant of FP-PEG-TAMRA labeling is 5.3 -fold, or $k_{\text {obs }} / k_{\text {cat }}$. Indeed, in the presence of excess prodrug (c.a. 3-times the $K_{M}$ ), we observed a 4-fold reduction in the FP-PEG-TAMRA binding rate constant $\left(k^{\prime}\right.$ obs $)$. Accordingly, the magnitude of substrate-competitive ABPP is highly 
dependent on the rate of ABPP probe inactivation $\left(k_{\text {inact }}\right)$, the concentration of ABPP probe, the substrate $K_{\mathrm{m}}$, the substrate $k_{c a t}$, the substrate concentration, and the probe reaction time.

\section{Comparison of mouse and human CES1 inhibitors}

WWL50 and WWL79 are potent inhibitors of mouse CES1, yet only WWL50 inhibits human CES1. Human and mouse CES1 are only 74\% similar in amino acid sequence, suggesting significant evolutionary drift and potentially divergent substrate profiles. In order to understand the mechanism of substrate specificity, we used the reported human CES1 crystal structure PDB ID: 2H7C) ${ }^{32}$ to build a homology model of mouse CES1 (UniProtKB ID: Q8VCC2). The covalent acylation products of WWL50 and 79 were modeled as adducts to the nucleophilic serine. Following minimization, the binding site volume of mouse CES1 appears significantly larger, suggesting access to a broader profile of substrates. While several residues contribute the volume increase, human Ile358 occupies more volume than mouse Leu358. Accordingly, the considerably larger alkyl morpholino group in WWL79 projects into the phenoxy binding site, occluding inhibitor binding (Figure 7B). In addition, mouse Arg460 is well positioned to solvate the WWL79 morpholino group through a bridging water molecule (Figure 7D), but the corresponding human Lys460 is oriented away from this region. Reported structure-activity relationship studies using phenol groups projecting into the alkylaminoacyl binding site led to a complete loss of human CES1 binding ${ }^{33}$, presumably because of a lack of solvation in a hydrophobic environment. Taken together these results suggest that the differences between human and mouse CES1s are due to hydrophobicity and binding site volume.

\section{DISCUSSION}

Identifying prodrug-activating enzymes is critical to understand the factors that contribute to drug delivery and drug efficacy, yet this analysis tends to occur late or as an afterthought in the drug development pipeline. This lag is partly a result of the typical late stage implementation of pro-moieties to modify biopharmaceutical properties and the timeconsuming biochemical separations and assays required for validation. However, with easy access to human and animal genomic and proteomic information, this process can be greatly accelerated. In this report, we describe a substrate-competitive ABPP approach to profile cellular serine hydrolases that bind a prodrug, and measure this binding through competition with broadly reactive chemical reporters targeting the serine hydrolase enzyme family. This method combines gel-based competitions with mass spectrometry protein identification, greatly accelerating the annotation of candidate prodrug binding enzymes. Further biochemical analysis is still required to confirm the enzymatic activity, and when available, selective inhibitors enable direct validation.

While this approach appears relatively straightforward, the time-dependent nature of the assay has some limitations. First, short ABPP probe reaction times are necessary to capture the kinetic window of substrate-mediated ABPP inhibition. Indeed, FP-PEG probes react exceptionally fast with CES1, making it experimentally challenging to capture the initial kinetic window of competition and surprisingly difficult to quantify by mass spectrometrybased competitive ABPP profiling. Unfortunately, this rapid competition may also prevent 
the detection of substrate saturation for many enzyme reactive sites, obscuring less abundant hydrolases and reducing the limit of detection. Second, the affinity and reactivity of distinct ABPP probes towards different enzymes exhibit variable kinetic parameters. Therefore, more exhaustive profiling may require a series of kinetically-tuned reactive probes with different enzyme preferences ${ }^{13}$, preferably with slower $k_{\text {inact }}$ rate constants. Finally, certain enzymes may interact with substrates via a competitive or mechanism-based mode of inhibition rather than catalyzing their turnover, thus requiring additional time-dependent analysis to distinguish substrate targets from true activating enzymes.

An alternative forward-genetics approach would involve overexpression or knockdown of all serine hydrolases for activity-guided assessment of the binding enzymes. Over-expressed enzymes could be tested without purification using the substrate-competitive ABPP methodology ${ }^{34}$. In addition, a set of generic serine hydrolase inhibitors could be examined, which in combination with ABPP methods, could quickly reduce the number of candidate activating enzymes. While our competitive analysis identified more than one oseltamivir binding hydrolase, CES1 inhibition alone accounted for $>95 \%$ of oseltamivir hydrolysis activity in intestine and liver derived cell lines. This suggests that oseltamivir is a mechanism-based inhibitor of at least one serine hydrolase (30 kD enzyme), although not an efficient substrate.

All together, substrate-based ABPP provided a simplified approach to gather initial data suggestive of the candidate oseltamivir-activating enzyme. While subsequent confirmation of enzymatic activity is required, substrate-competitive ABPP provides a broad rapid survey of candidate prodrug activating enzymes. We anticipate this approach could be applied to annotate other uncharacterized prodrug activating enzymes, providing a preliminary screen to highlight candidate enzymes. Finally, using gel-based and mass spectrometry competitive ABPP approaches, we validate the species-specific inhibition profile of WWL50 in Caco-2 and HepG2 cells, establishing a useful new probe for mechanistic studies on CES1-activated ester prodrugs.

\section{Supplementary Material}

Refer to Web version on PubMed Central for supplementary material.

\section{ACKNOWLEDGMENTS}

We would like to thank Drs. Chester Provoda, Kefeng Sun, and Yasuhiro Tsume for experimental assistance and advice. Financial support is provided by the National Institutes of Health R01 GM037188 (G.L.A. and B.R.M.), R00 CA151460 (B.R.M.), DP2 GM114848 (B.R.M.), R01 GM065372 (P.G. and H.A.C), the American Heart Association 14POST20420040 (J.D.M.), and the University of Michigan.

\section{ABBREVIATIONS}

$\begin{array}{ll}\text { ABPP } & \text { activity-based protein profiling } \\ \text { FP } & \text { fluorophosphonate }\end{array}$




\begin{tabular}{|c|c|}
\hline FP-PEG-TAMRA & $\begin{array}{l}\text { fluorophosphonate-polyethylene glycol- } \\
\text { carboxytetramethylrhodamine }\end{array}$ \\
\hline CESs & carboxylesterases \\
\hline SHs & serine hydrolases \\
\hline ABPP-SILAC & $\begin{array}{l}\text { competitive ABPP with stable isotope labeling by amino acids in } \\
\text { cell culture }\end{array}$ \\
\hline HepG2 & human hepatocellular carcinoma \\
\hline Caco-2 & human colorectal adenocarcinoma \\
\hline SDS-PAGE & sodium dodecyl sulfate-polyacrylamide gel electrophoresis \\
\hline DMEM & dulbecco's modified eagle medium \\
\hline FBS & fetal bovine serum \\
\hline DTT & dithiothreitol \\
\hline ESI & electrospray ionization \\
\hline IMS & ion mobility separation \\
\hline $\mathbf{C E}$ & collision energy \\
\hline FDR & false discovery rate \\
\hline SEM & standard error of mean \\
\hline
\end{tabular}

\section{References}

1. Hosokawa M, Maki T, Satoh T. Characterization of molecular species of liver microsomal carboxylesterases of several animal species and humans. Arch Biochem Biophys. 1990; 277(2): 219-27. [PubMed: 2310190]

2. Long JZ, Cravatt BF. The metabolic serine hydrolases and their functions in mammalian physiology and disease. Chem Rev. 2011; 111(10):6022-63. [PubMed: 21696217]

3. Simon GM, Cravatt BF. Activity-based proteomics of enzyme superfamilies: serine hydrolases as a case study. J Biol Chem. 2010; 285(15):11051-5. [PubMed: 20147750]

4. Kim I, Chu XY, Kim S, Provoda CJ, Lee KD, Amidon GL. Identification of a human valacyclovirase: biphenyl hydrolase-like protein as valacyclovir hydrolase. J Biol Chem. 2003; 278(28):25348-56. [PubMed: 12732646]

5. Laizure SC, Parker RB, Herring VL, Hu ZY. Identification of carboxylesterase-dependent dabigatran etexilate hydrolysis. Drug Metab Dispos. 2014; 42(2):201-6. [PubMed: 24212379]

6. Hauel NH, Nar H, Priepke H, Ries U, Stassen JM, Wienen W. Structure-based design of novel potent nonpeptide thrombin inhibitors. J Med Chem. 2002; 45(9):1757-66. [PubMed: 11960487]

7. Cravatt BF, Wright AT, Kozarich JW. Activity-based protein profiling: from enzyme chemistry to proteomic chemistry. Annu Rev Biochem. 2008; 77383-414

8. Bachovchin DA, Cravatt BF. The pharmacological landscape and therapeutic potential of serine hydrolases. Nat Rev Drug Discov. 2012; 11(1):52-68. [PubMed: 22212679]

9. Sanman LE, Bogyo M. Activity-based profiling of proteases. Annu Rev Biochem. 2014; 83249-73

10. Patricelli MP, Szardenings AK, Liyanage M, Nomanbhoy TK, Wu M, Weissig H, Aban A, Chun D, Tanner S, Kozarich JW. Functional interrogation of the kinome using nucleotide acyl phosphates. Biochemistry. 2007; 46(2):350-8. [PubMed: 17209545] 
11. Leung D, Hardouin C, Boger DL, Cravatt BF. Discovering potent and selective reversible inhibitors of enzymes in complex proteomes. Nat Biotechnol. 2003; 21(6):687-91. [PubMed: 12740587]

12. Long JZ, Li W, Booker L, Burston JJ, Kinsey SG, Schlosburg JE, Pavon FJ, Serrano AM, Selley DE, Parsons LH, Lichtman AH, Cravatt BF. Selective blockade of 2-arachidonoylglycerol hydrolysis produces cannabinoid behavioral effects. Nat Chem Biol. 2009; 5(1):37-44. [PubMed: 19029917]

13. Adibekian A, Martin BR, Chang JW, Hsu KL, Tsuboi K, Bachovchin DA, Speers AE, Brown SJ, Spicer T, Fernandez-Vega V, Ferguson J, Hodder PS, Rosen H, Cravatt BF. Confirming target engagement for reversible inhibitors in vivo by kinetically tuned activity-based probes. J Am Chem Soc. 2012; 134(25):10345-8. [PubMed: 22690931]

14. Xu H, Sabit H, Amidon GL, Showalter HD. An improved synthesis of a fluorophosphonatepolyethylene glycol-biotin probe and its use against competitive substrates. Beilstein J Org Chem. 2013; 989-96

15. Shi D, Yang J, Yang D, LeCluyse EL, Black C, You L, Akhlaghi F, Yan B. Anti-influenza prodrug oseltamivir is activated by carboxylesterase human carboxylesterase 1 , and the activation is inhibited by antiplatelet agent clopidogrel. J Pharmacol Exp Ther. 2006; 319(3):1477-84. [PubMed: 16966469]

16. Zhu HJ, Wang X, Gawronski BE, Brinda BJ, Angiolillo DJ, Markowitz JS. Carboxylesterase 1 as a determinant of clopidogrel metabolism and activation. J Pharmacol Exp Ther. 2013; 344(3):66572. [PubMed: 23275066]

17. Hosokawa M. Structure and catalytic properties of carboxylesterase isozymes involved in metabolic activation of prodrugs. Molecules. 2008; 13(2):412-31. [PubMed: 18305428]

18. Fleming CD, Bencharit S, Edwards CC, Hyatt JL, Tsurkan L, Bai F, Fraga C, Morton CL, HowardWilliams EL, Potter PM, Redinbo MR. Structural insights into drug processing by human carboxylesterase 1: tamoxifen, mevastatin, and inhibition by benzil. J Mol Biol. 2005; 352(1):16577. [PubMed: 16081098]

19. Holmes RS, Wright MW, Laulederkind SJ, Cox LA, Hosokawa M, Imai T, Ishibashi S, Lehner R, Miyazaki M, Perkins EJ, Potter PM, Redinbo MR, Robert J, Satoh T, Yamashita T, Yan B, Yokoi T, Zechner R, Maltais LJ. Recommended nomenclature for five mammalian carboxylesterase gene families: human, mouse, and rat genes and proteins. Mamm Genome. 2010; 21(9-10):427-41. [PubMed: 20931200]

20. Ross MK, Borazjani A, Wang R, Crow JA, Xie S. Examination of the carboxylesterase phenotype in human liver. Arch Biochem Biophys. 2012; 522(1):44-56. [PubMed: 22525521]

21. Bachovchin DA, Ji T, Li W, Simon GM, Blankman JL, Adibekian A, Hoover H, Niessen S, Cravatt BF. Superfamily-wide portrait of serine hydrolase inhibition achieved by library-versuslibrary screening. Proc Natl Acad Sci U S A. 2010; 107(49):20941-6. [PubMed: 21084632]

22. Kidd D, Liu Y, Cravatt BF. Profiling serine hydrolase activities in complex proteomes. Biochemistry. 2001; 40(13):4005-15. [PubMed: 11300781]

23. Jacobson MP, Pincus DL, Rapp CS, Day TJ, Honig B, Shaw DE, Friesner RA. A hierarchical approach to all-atom protein loop prediction. Proteins. 2004; 55(2):351-67. [PubMed: 15048827]

24. Jacobson MP, Friesner RA, Xiang Z, Honig B. On the role of the crystal environment in determining protein side-chain conformations. J Mol Biol. 2002; 320(3):597-608. [PubMed: 12096912]

25. Ho BK, Gruswitz F. HOLLOW: generating accurate representations of channel and interior surfaces in molecular structures. BMC Struct Biol. 2008; 849

26. McClellan K, Perry CM. Oseltamivir: a review of its use in influenza. Drugs. 2001; 61(2):263-83. [PubMed: 11270942]

27. Suzaki Y, Uemura N, Takada M, Ohyama T, Itohda A, Morimoto T, Imai H, Hamasaki H, Inano A, Hosokawa M, Tateishi M, Ohashi K. The effect of carboxylesterase 1 (CES1) polymorphisms on the pharmacokinetics of oseltamivir in humans. Eur J Clin Pharmacol. 2013; 69(1):21-30. [PubMed: 22673926] 
28. Imai T, Imoto M, Sakamoto H, Hashimoto M. Identification of esterases expressed in Caco-2 cells and effects of their hydrolyzing activity in predicting human intestinal absorption. Drug Metab Dispos. 2005; 33(8):1185-90. [PubMed: 15908471]

29. Hidalgo IJ, Raub TJ, Borchardt RT. Characterization of the human colon carcinoma cell line (Caco-2) as a model system for intestinal epithelial permeability. Gastroenterology. 1989; 96(3): 736-49. [PubMed: 2914637]

30. Adibekian A, Martin BR, Wang C, Hsu KL, Bachovchin DA, Niessen S, Hoover H, Cravatt BF. Click-generated triazole ureas as ultrapotent in vivo-active serine hydrolase inhibitors. Nat Chem Biol. 2011; 7(7):469-78. [PubMed: 21572424]

31. Bar-Even A, Noor E, Savir Y, Liebermeister W, Davidi D, Tawfik DS, Milo R. The moderately efficient enzyme: evolutionary and physicochemical trends shaping enzyme parameters. Biochemistry. 2011; 50(21):4402-10. [PubMed: 21506553]

32. Bencharit S, Edwards CC, Morton CL, Howard-Williams EL, Kuhn P, Potter PM, Redinbo MR. Multisite promiscuity in the processing of endogenous substrates by human carboxylesterase 1 . J Mol Biol. 2006; 363(1):201-14. [PubMed: 16962139]

33. Wadkins RM, Hyatt JL, Wei X, Yoon KJ, Wierdl M, Edwards CC, Morton CL, Obenauer JC, Damodaran K, Beroza P, Danks MK, Potter PM. Identification and characterization of novel benzil (diphenylethane-1,2-dione) analogues as inhibitors of mammalian carboxylesterases. J Med Chem. 2005; 48(8):2906-15. [PubMed: 15828829]

34. Blankman JL, Simon GM, Cravatt BF. A comprehensive profile of brain enzymes that hydrolyze the endocannabinoid 2-arachidonoylglycerol. Chem Biol. 2007; 14(12):1347-56. [PubMed: 18096503] 
A

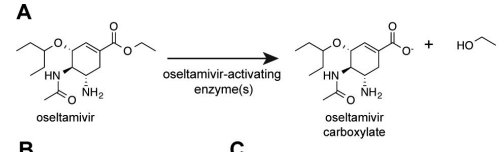

B

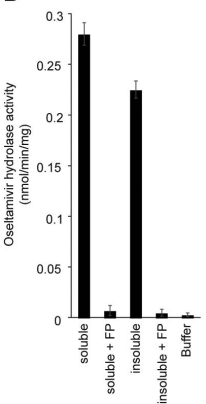

Soluble
FP-PEG-TAMRA $(0.5)$

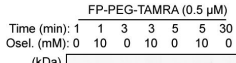

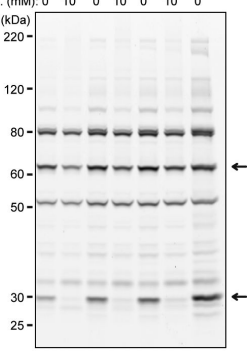

D

E
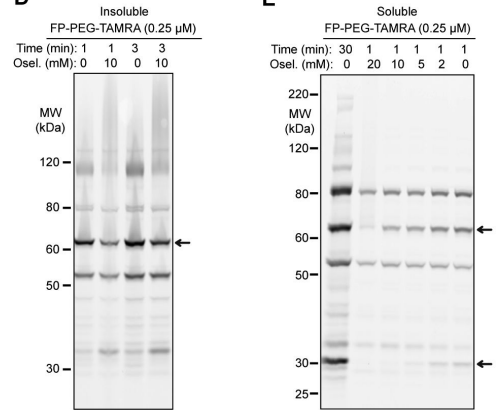

Figure 1. Competitive activity-based profiling of serine hydrolase activities in in Caco-2 homogenates

(A) Activating esterases hydrolyze oseltamivir to release active oseltamivir carboxylate. (B) Oseltamivir hydrolysis is blocked in Caco-2 homogenates after fluorophosphonate (FP) treatment. (C) Time-dependent oseltamivir competition in soluble Caco-2 homogenates. (D) Time-dependent oseltamivir competition in insoluble Caco-2 homogenates. (E) Higher substrate concentrations are more efficient at fluorophosphonate competition. 
A

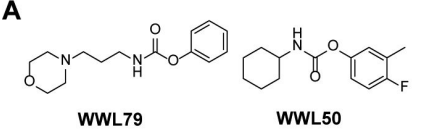

B

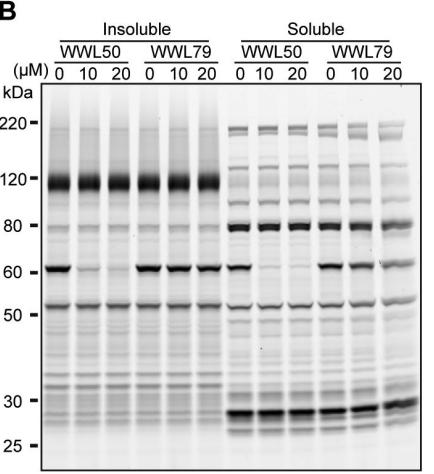

C

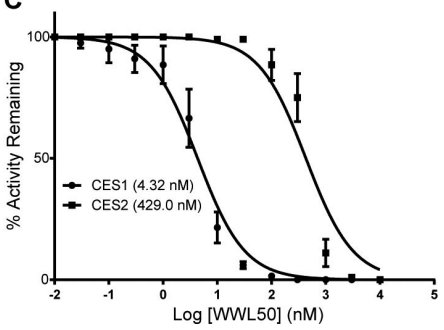

Figure 2. Human CES1 inhibition by WWL50

(A) Chemical structures of WWL50 and WWL79. (B) FP-PEG-TAMRA competitive activity-based profiling of WWL50 in human Caco-2 homogenates highlights selective inhibition. (C) WWL50 is 100x more potent for recombinant human CES1 than recombinant human CES2. Error bars represented as standard deviations of 3-4 individual experiments. 


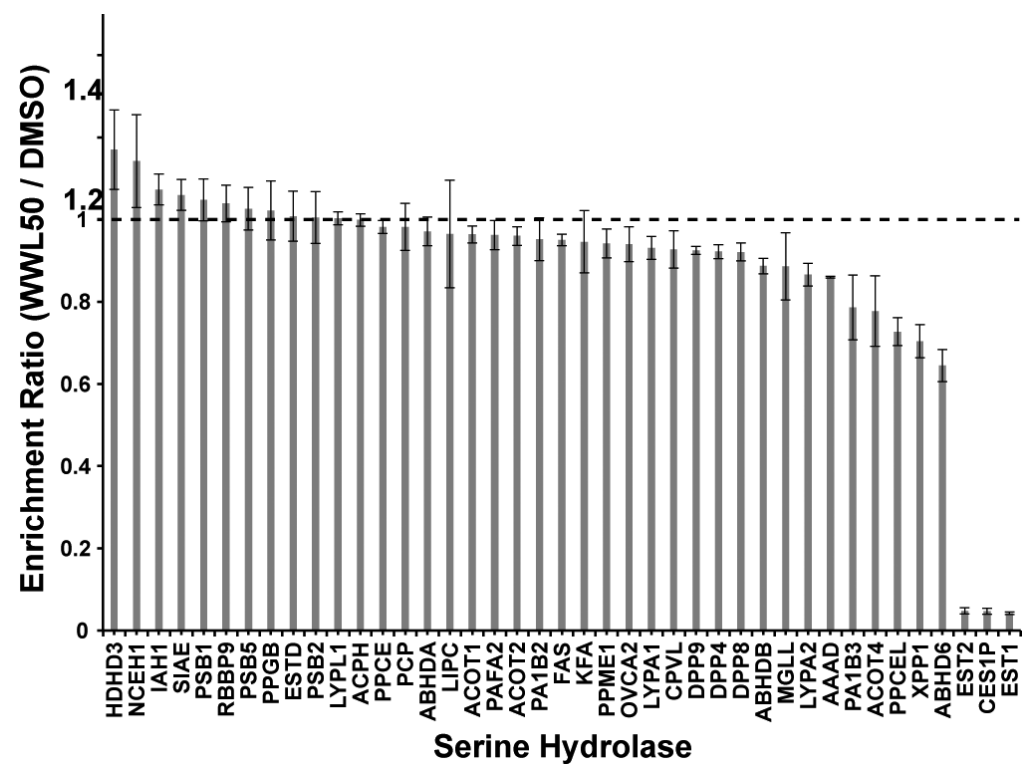

Figure 3. Identification of WWL50 targets in Caco-2 homogenates by ABPP-SILAC

Error bars represent SILAC ratio standard error quantified from tryptic peptides pooled from both soluble and membrane proteomes for two independent biological replicates. EST1 and EST2 are the human identifiers for CES1 and CES2, respectively. 


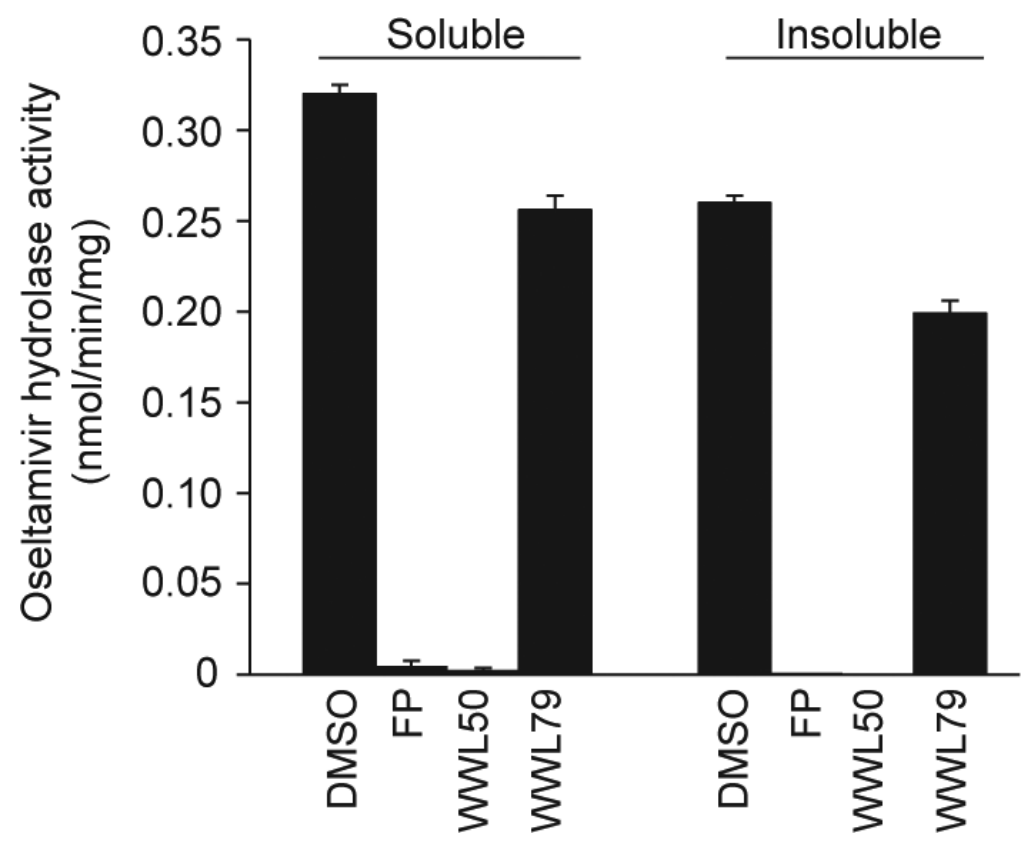

Figure 4. Inhibition of oseltamivir hydrolysis with CES1 inhibitors in Caco-2 homogenates Results of time-dependent oseltamivir hydrolysis are shown as mean \pm SEM. 

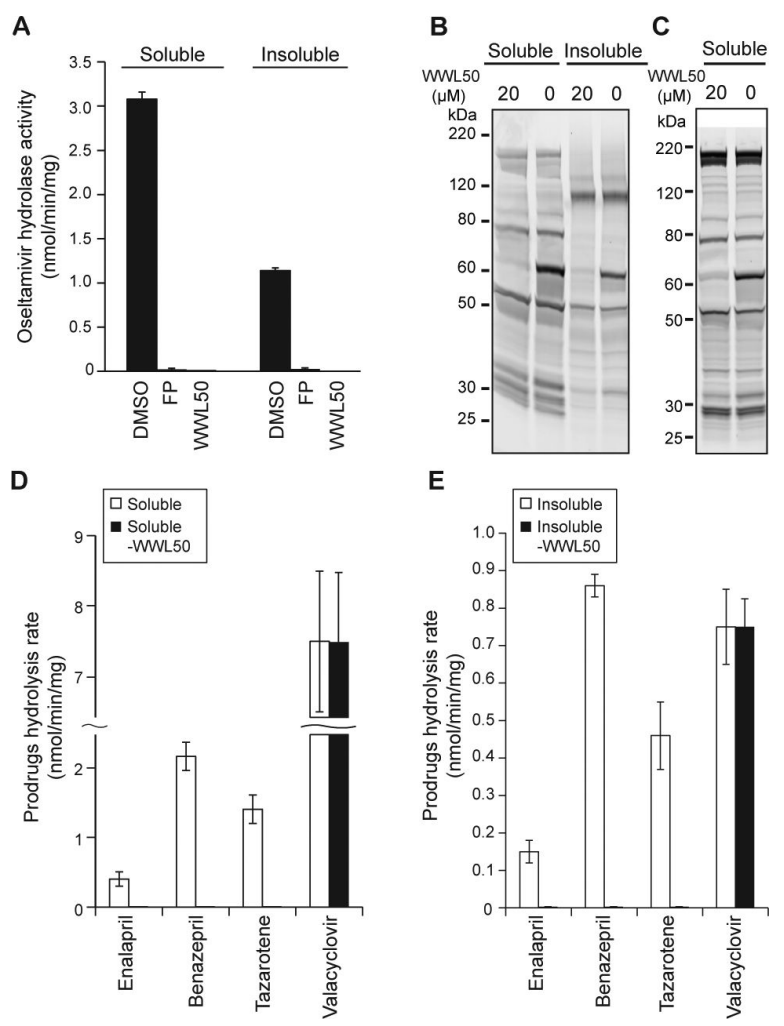

Figure 5. Ester prodrug hydrolysis in human HepG2 liver cell homogenates

(A) Fluorophosphonates and WWL50 block oseltamivir hydrolysis in HepG2 soluble and insoluble homogenates. (B) WWL50 blocks FP-PEG-TAMRA labeling of a $60 \mathrm{kD}$ serine hydrolase in soluble and insoluble homogenates from HepG2 cells, corresponding to CES1. (C) FP-TAMRA, which replaces the PEG linker with an aliphatic linker, shows similar competition for a $60 \mathrm{kD}$ serine hydrolase (CES1). (D) WWL50 blocks ethyl ester hydrolysis activity in HepG2 soluble homogenates. (E) Insoluble homogenates have reduced ethyl ester hydrolysis, but essentially all activity is blocked by WWL50. Enalapril (0.5 mM), benazapril $(0.5 \mathrm{mM})$, tazarotene $(0.25 \mathrm{mM})$ and valacyclovir $(0.5 \mathrm{mM})$ were hydrolyzed at $37^{\circ} \mathrm{C}$ in HepG2 cytosol $(1 \mathrm{mg} / \mathrm{ml})$ followed by quantitative HPLC analysis at different time points. Results are represented as the average \pm SD of 2-3 individual experiments. 
A

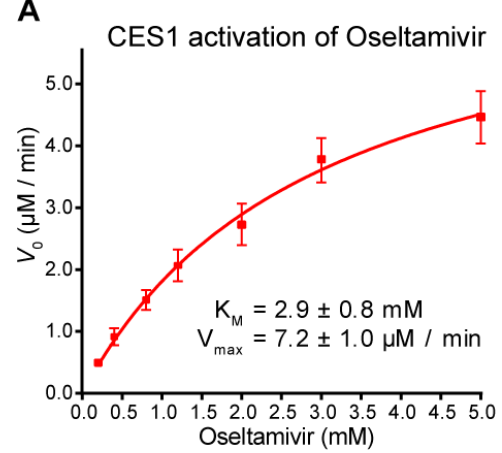

B

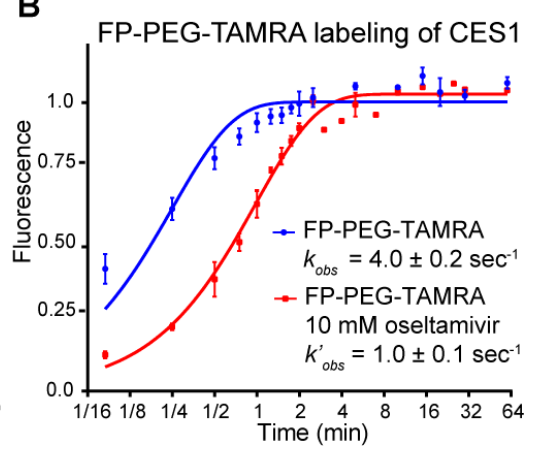

Figure 6. Oseltamivir impedes the inactivation rate of CES1 by fluorophosphonates (A) Substrate-dependent velocity of recombinant human CES1-catalyzed oseltamivir hydrolysis and associated steady-state kinetic parameters. (B) Time-dependent fluorophosphonate inactivation of recombinant human CES1 and the associated pseudofirst-order rate constant. Near-saturating oseltamivir concentrations lead to a 4-fold reduction in the observed rate constant for fluorophosphonate inactivation of CES1. Error bars represent the standard deviation from 3-4 replicates. 


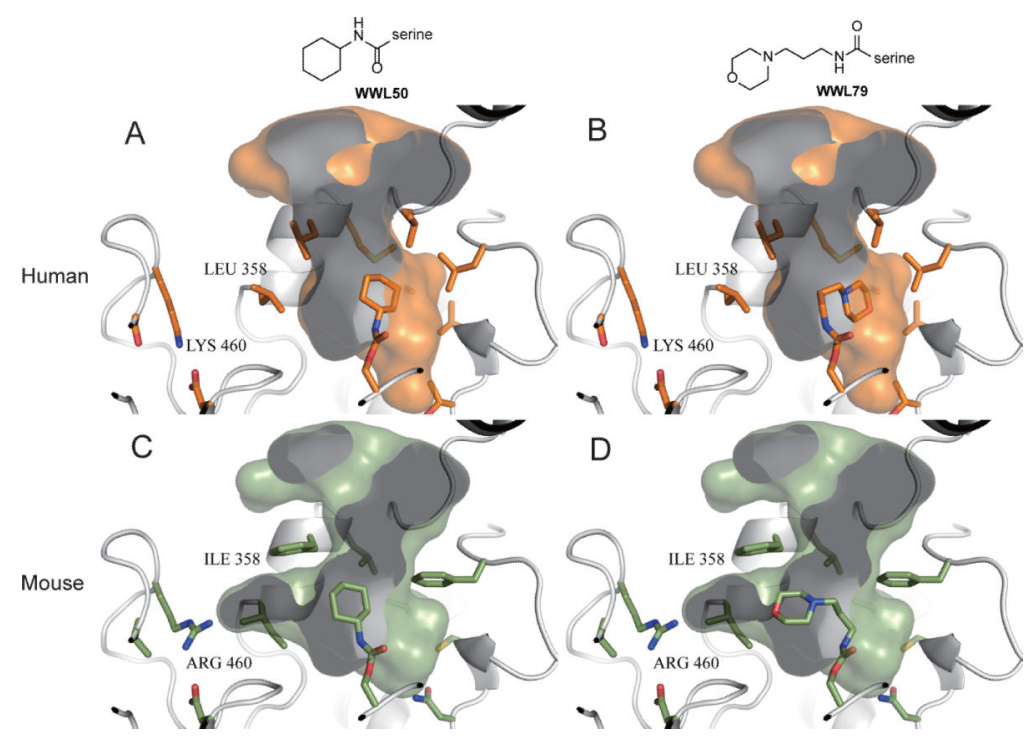

Figure 7. Covalent docking of WWL50 and 79 to human and mouse CES1

WWL50 and WWL79 were covalently docked to the catalytic Serine 221 in the crystal structure of human CES1 (PDB ID: 2H7C, colored orange) and the homology model of mouse CES1 (colored green). The active site rendered as surface was clipped to show the difference in alkylaminoacyl binding site volume across human and mouse CES1s. WWL50 and 79 along with residues that differ between the human and mouse CES1 enzymes are shown as sticks. (A) WWL50 docked into the human CES1. (B) WWL79 docked into the human CES1. (C) WWL50 docked into to the mouse CES1 homology model. (D) WWL79 docked into the mouse CES1 homology model. 